\title{
An Improved Assay for Measuring Low Levels of Nitric Oxide in Cultured Pulmonary Myofibroblasts
}

\author{
Bethel V. Sharma1, Naomi S. Rowland2, Margaret M. Clouse², Nancy A. Rice ${ }^{2 *}$ \\ ${ }^{1}$ Department of Chemistry, Sewanee: The University of the South, Sewanee, TN, USA \\ ${ }^{2}$ Department of Biology, Western Kentucky University, Bowling Green, KY, USA \\ Email: "nancy.rice@wku.edu
}

Received 28 February 2014; revised 3 April 2014; accepted 11 April 2014

Copyright (C) 2014 by authors and Scientific Research Publishing Inc.

This work is licensed under the Creative Commons Attribution International License (CC BY). http://creativecommons.org/licenses/by/4.0/

(c) (i) Open Access

\begin{abstract}
Quantification of nitric oxide (NO) from cultured cells is a valuable tool for studying cell signaling. Detection of NO in biological fluids can be difficult however, due to its transient half-life and low physiological concentrations. In this study, we have refined an existing amperometric method to determine relative levels of accumulated nitrogen oxides $\left(\mathrm{NO}_{\mathrm{x}}\right)$ in cell culture and have used this method to reproducibly quantify NO from cultured pulmonary myofibroblasts. Basal levels of NO produced by pulmonary myofibroblasts ranged from $0.6 \mathrm{nM}$ to $20 \mathrm{nM}$ and varied due to the growth conditions of the cells, i.e. higher NO concentrations were observed in differentiated cells. The constitutive eNOS isoform is primarily responsible for the observed NO accumulation in these cells since transcript levels of eNOS are 10-fold higher than the inducible iNOS form while nNOS was undetectable. Treatment of myofibroblasts with the inhibitors L-NNA and L-NAME resulted in a concentration dependent decrease in measured NOx. Overall, the improved assay presented here should be applicable to measuring $\mathrm{NO}_{\mathrm{x}}$ levels from many different cell types and under a wide variety of conditions.
\end{abstract}

\section{Keywords}

Myofibroblast, Pulmonary, Nitric Oxide, eNOS

\section{Introduction}

The origin of myofibroblasts in the lung is not clear, although the favored model is that resident adventitial fi-

*Corresponding author. 
broblasts differentiate in response to multiple paracrine-mediated pathways and, as a result, undergo the phenotypic changes associated with myofibroblasts, i.e. increased extracellular matrix deposition and increased contractility (reviewed in [1]-[7]). Various signaling pathways, including the nitric oxide (NO) pathway, have been implicated in the process of fibroblast differentiation into myofibroblasts. NO is free radical gas produced from the enzymatic conversion of L-arginine to L-citrulline by NO synthases (NOSs) (reviewed in [8]). NO subsequently binds to the active site heme of guanylyl cyclase, resulting in cGMP formation and ultimately stimulating cGMP-dependent protein kinases [9]. Three different forms of NOS have been identified and named based upon tissue of origin: neural NOS (nNOS), inducible NOS (iNOS), and endothelial NOS (eNOS), although it is now known that many cells types express multiple isoforms [10]. Both nNOS and eNOS are constitutive, $\mathrm{Ca}^{2+}-$ dependent enzymes, which produce NO in the pM range, while the activity of iNOS is induced in response to activated macrophages, is $\mathrm{Ca}^{2+}$-independent, and results in nM levels of NO [11]. While constitutively produced NO is most commonly associated with maintenance of vascular tone in the endothelium [12], it is also implicated as a key modulator of myofibroblast cell growth and activity in numerous tissues including lung [2] [13], liver [14], kidney [15], heart [16] [17], and urogential [18] tissues.

The ability to measure physiologically relevant levels of NO is an important research tool, particularly given recent reports that constitutively produced NO concentrations in vivo are likely much lower than once presumed [19]. Current methods available for detecting biologically produced NO include spectrophotometric, fluorometric, electrochemical, chemiluminescent, and electromagnetic spin resonance techniques [20]. Of these analyses however, only fluorometric and electrochemical have both the sensitivity and flexibility to measure low levels of constitutively produced NO from cultured cells in situ. Particularly, the fluorescent indicator diaminofluorescein (DAF) has been used to quantify NO generated by cultured myofibroblasts [21] [22]. While this method is highly sensitive, it has had limited success due to the susceptibility of the calibration standard to photolysis. Other studies have reported an amperometric technique to measure NO using a specific constant voltage electrode, the inNO-T Nitric Oxide Measuring System, in a manner similar to the one presented here [23] [24]. This electrochemical method is both sensitive and affordable although it does have intrinsic limitations due to the conversion of nitrite to nitrate/nitric acid in aqueous solution which is undetectable by the electrode, ultimately resulting in signal loss. Both the fluorometric and amperometric approaches directly quantify NO from the cell culture media which is known to contain measurable amounts of nitrate [23]. Therefore, the goal of this study was to refine the electrode technique to both increase sensitivity and avoid loss of signal due to nitrate formation in aqueous medium in order to further investigate the role of NO signaling pathways in pulmonary myofibroblasts. Although this study was designed to quantify NO released from myofibroblasts, the simple and reproducible method of monitoring indirect NO presented here is broadly applicable to many other basal-level NO producing cells.

\section{Materials and Methods}

\subsection{Reagents}

All buffers and chemicals were cell culture grade and purchased from Sigma Chemical Company (St. Louis, MO). The NOS inhibitors, N-omega-nitro-L-arginine methyl ester hydrochloride (L-NAME) and N-omega-nitroL-arginine (L-NNA) were purchased from EMD Millipore (Billerica, MA).

\subsection{Cell Line Preparation}

In this study, pulmonary fibroblasts were isolated from 28 - 33 day old female Lewis rats as previously described [25]. Cells were cultured in DMEM (Gibco BRL, Carlsbad, CA) supplemented with $10 \%$ fetal bovine serum (FBS) (Hyclone, Logan, UT), $100 \mathrm{U}$ penicillin, $100 \mu \mathrm{g} / \mathrm{ml}$ streptomycin, $2 \mathrm{mM}$ L-glutamine, and $20 \mathrm{mM}$ HEPES, pH 7.55 (Gibco BRL). For routine passaging and maintenance, cells were grown in serum-supplemented $(10 \% \mathrm{v} / \mathrm{v})$ media in T-75 cell culture flasks from Corning (Tewksbury, MA). It is well established that isolated fibroblasts cultured on a plastic substrate display a myofibroblast phenotype as determined by $\alpha$-smooth muscle actin expression [3] [26]. Cultured myofibroblasts are known to differentiate under conditions of serum starvation (when growth factors are lacking in the cell culture media) as verified by skeletal muscle protein expression [27]. 


\subsection{Culture Conditions}

Myofibroblasts were seeded at a density of $1 \times 10^{5}$ cells/well, in 6-well plates, and grown for approximately 48 h or until $\sim 75 \%$ confluent. Subsequently, the growth media was changed to either fresh $10 \%$ FBS (v/v) or $0 \%$ (serum-free) DMEM plus either of the NOS inhibitors L-NAME or L-NNA at various concentrations; cells were incubated overnight prior to assay.

Approximately $16 \mathrm{~h}$ later, the growth media was aspirated from each well and replaced with phosphate buffered saline (PBS), pH 7.4, containing $100 \mu \mathrm{M} \mathrm{CaCl}_{2}, 1 \mathrm{mM}$ l-arginine, and $1 \mu \mathrm{M}$ A23187, similar to the method of Leikert et al. [22]. The growth media was removed to avoid interference from serum nitrate as previously reported [23]. Cells were incubated for $3 \mathrm{~h}$ at room temperature. This time point was selected after several pilot experiments that determined 1 - 2 h was not long enough for sufficient $\mathrm{NO}_{\mathrm{X}}$ accumulation, yet by $4 \mathrm{~h}$ of incubation the myofibroblasts had begun to lift off the plate (data not shown). Subsequently, $900 \mu \mathrm{L}$ from each sample was removed and combined with $100 \mu \mathrm{L}$ of $1 \mathrm{M}$ Tris buffer, pH 9.2, in a 24-well plate. Samples were incubated with a nitrate reductor (NR) wire for 15 min with stirring to reduce any accumulated nitrate back to nitrite. The NR wire is $3 \mathrm{~cm}$ long $\times 3 \mathrm{~mm}$ wide and is made from a metal alloy that catalyzes the reduction of nitrate to nitrite, which is subsequently detected by the electrode (Harvard apparatus). The NR wires were stored in $0.01 \mathrm{M} \mathrm{H}_{2} \mathrm{SO}_{4}$ when not in use, and were rinsed with deionized water prior to placing into the well with the sample solution.

\subsection{NO Detection Assay}

The NO selective electrode (Innovative Instruments, Tampa, FL) was equilibrated in a 20 mL acid-iodide bath (20 mM potassium iodide, $100 \mathrm{mM}$ sulfuric acid) with constant stirring at room temperature for 30 min prior to calibration. A standard curve in picoamps (pA) was subsequently generated by measuring various known concentrations of sodium nitrite added to the bath. The peak currents were monitored and used to record an amperogram using the inNO-T software v3.2 provided by Innovative Instruments. To measure extracellular $\mathrm{NO}_{\mathrm{X}}, 100$ uL from each NR treated sample was added to the acid iodide bath in which the electrode was immersed. Individual peaks were resolved on the amperogram within 3 - 5 minutes. The signal was allowed to return to baseline before subsequent sample additions. Data points were collected by subtracting the initial baseline from the maximum peak height value for each sample. The $\mathrm{NO}_{\mathrm{X}}$ concentration for each sample was extrapolated from a standard calibration curve prepared prior to each experiment.

\subsection{Reverse Transcription PCR}

Pulmonary myofibroblasts were grown in culture as described above in 2.1 in either media containing $10 \%$ FBS or 0\% FBS. After 48 hours, cells were harvested in PBS and suspended in TRIzol reagent (Invitrogen). Total RNA was isolated according to the manufacturer's protocol (TRIzol). One $\mu \mathrm{g}$ total RNA was converted to cDNA by the High Capacity cDNA archive kit (Applied Biosystems). Exon-exon boundaries in mRNA sequences for NOS1 [Rn00583793mi], NOS2 [Rn00561646mi], or NOS3 [Rn 02132634si] were identified and submitted to Applied Biosystems, Inc. for Taqman gene expression assay design. Each primer set was chosen so that amplicons would cross exon boundaries to prevent contamination with genomic DNA. Derived experimental Ct values were converted to copy number using a standard curve and subsequently normalized to an endogenous control, $\beta$-glucuronidase (GUS) [Rn00566655mi].

\section{Results}

\subsection{Nanomolar Concentrations of NO Are Produced by Pulmonary Myofibroblasts}

It is known that pulmonary myofibroblasts in culture alter their biological function and genetic signature significantly in response serum concentration changes [27]. Therefore, for any assay to be physiologically applicable to myofibroblasts, NO must be quantifiable in media with different serum amounts. When known concentrations of nitrite, first converted to NO in an acid-iodide bath, were measured in culture media that contained either $10 \%$ or $0 \%$ FBS, using an NO-selective electrode coated with a gas permeable membrane (the amiNO-700 sensor), non-overlapping calibration curves resulted (Figure 1). Although the coefficient of determination $\left(R^{2}\right)$ for both samples was $>0.90$, the resulting linear regressions produced vastly different calibration curves with equations 


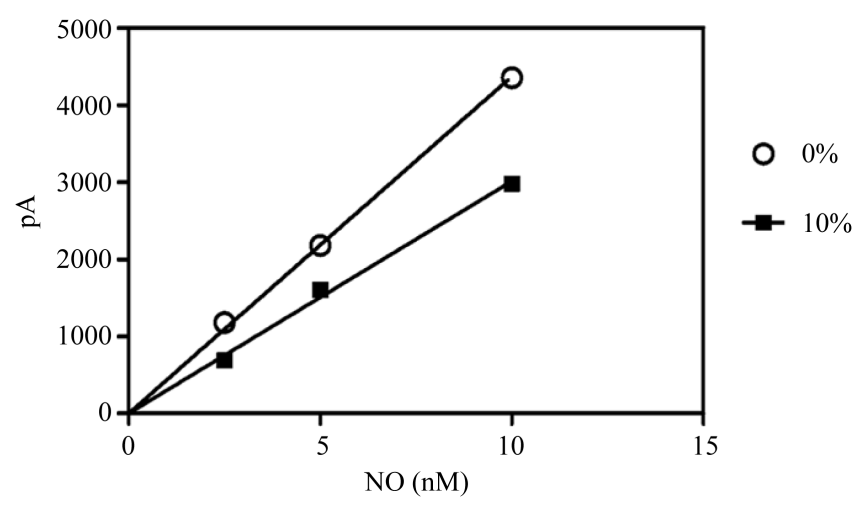

Figure 1. Calibration curves for the selective NO electrode in the presence of different serum concentrations. A simple linear regression for a typical three-point calibration curve is shown in media with and without FBS. Results are given as the mean \pm 1 SD (error bars obscured by data points).

of $\mathrm{y}=502.7 \mathrm{x}$ and $\mathrm{y}=335.7 \mathrm{x}$ for the serum free media and media containing $10 \% \mathrm{FBS}$, respectively. To overcome this dilemma, we tested whether NO could be accurately measured in PBS alone. As shown in Figure 2(a) and Figure 2(b), NO is indeed readily detectable by the amiNO-700 sensor in PBS. Therefore, the ability to electrochemically quantify NO production in PBS versus media greatly increases the efficiency of the assay by eliminating the need for multiple media-dependent calibration steps, while also preventing any potential media-induced matrix effects.

The inNO-T Nitric Oxide Measuring System, in addition to the amiNO-700 sensor, contains proprietary nitrate reductor (NR) wires comprised of a reusable metallic catalyst that reduces any nitrate in the samples back to nitrite prior to analysis. Although NO released from the cells is readily detectable using the system, when the nitrate reduction step was added to the protocol, a two-fold amplification in NO was measured (Figure 2(c)). Thus, the simple wire-based reduction step also serves to improve the amperometric assay by increasing its sensitivity and ease; this is in contrast to intricate enzymatic reduction methods previously used with electrochemical probes [23] [24].

\subsection{The Most Abundant NOS Isoform in Pulmonary Myofibroblasts Is eNOS}

In order to properly use the bioassay in experimental design, it is essential to know which of the three NOS isoforms are expressed in pulmonary myofibroblasts, in particular, since the inducible NOS isozyme has significantly different pharmacokinetic properties and can produce $\sim 1000$ times the molar concentration of NO when compared to either constitutive form [19]. When the amount of each NOS mRNA was quantified from cultured pulmonary myofibroblasts, from both $10 \%$ FBS and serum free media, the most abundantly expressed isoform was eNOS, regardless of serum, followed by significantly lower levels ( 10-fold) of iNOS mRNA; nNOS levels were undetectable (Figure 3). Transcriptional changes of these two NOS variants was reciprocal in that eNOS increased under normal growth conditions, whereas iNOS expression increased when cells were differentiated by the removal of serum. The serum-mediated differential expression implies there may be distinct roles for each isoform requiring different molar concentrations of NO.

\subsection{Serum Deprivation Increases Basal NO Production in Pulmonary Myofibroblasts}

When the enhanced assay was used to quantify constitutively produced NO in pulmonary myofibroblasts under both normal growth conditions ( $10 \%$ serum) and after differentiation ( $0 \%$ serum), the assay proved to be reproducible and sensitive; differentiated cells produced $\sim 30$-fold more NO under basal conditions than those growing in serum ( 20 nM vs $\sim 0.60 \mathrm{nM}$ ) (Figure 4(b) and Figure 4(c)). Moreover, treatment of the cells with the constitutive NOS selective inhibitors L-NNA and its methyl ester, L-NAME, dose-dependently reduced NO release from differentiated cells, while proliferating cells were not responsive to either inhibitor (Figure 4(b) and Figure 4(c)) [28]. The variance in the inhibitor response to serum may reflect changes in eNOS subcellular 
(a)

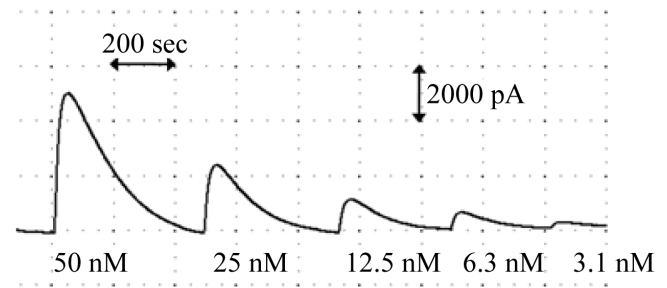

(b)

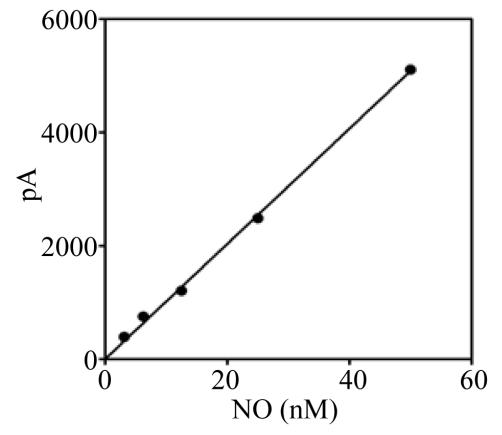

(c)

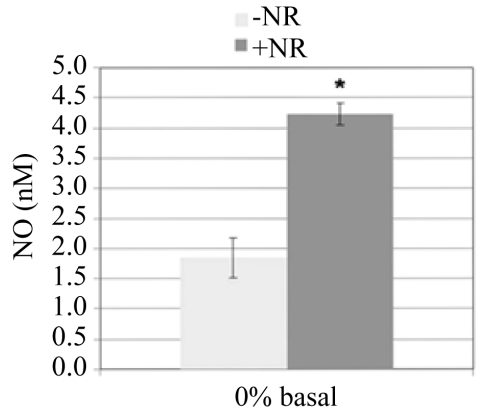

Figure 2. NO quantification in PBS with and without nitrite reduction. (a) A typical amperogram used for calibration of the sensor. The signal obtained by addition of nitrite standards to the acid iodide bath determines the measured peak heights used for calibration; (b) A typical five-point calibration curve for nitrite standards diluted in PBS using the data obtained in (a). The equation for the standard curve is $\mathrm{y}=101.68 \mathrm{x}$ and $R^{2}=0.9985$; (c) Comparison of NOx signals from serum-free (0\%) myofibroblast samples that were and were not incubated with the NR wire for 15 min prior to electrochemical analysis results in a 2-fold increase in signal. Results are given as the mean \pm SEM $(n=3)$; An * indicates significance ( $p$ $<0.05$ ) as determined by paired $t$-test.

location since in other cell types, cellular locale strongly affects eNOS' ability to produce NO and respond to $\mathrm{Ca}^{2+}$ [29]. The fact that NO production was not completely abolished in the presence of either inhibitor however, does suggest that iNOS, while expressed 10-fold less than eNOS, contributes somewhat to the overall formation of NO in pulmonary myofibroblasts.

\section{Discussion}

In this report we describe an enhanced assay that provides a straightforward method for measuring relative changes of NO produced by cultured pulmonary myofibroblasts. The method utilizes a commercially available measurement system with nitrate reduction capabilities and is also adaptable for measuring NO in many different cultured cell types. While the inNO-T Nitric Oxide Measuring System has been reported as a sensitive NO detection platform, previous studies included an enzymatic reduction step that is time-consuming and dependent 

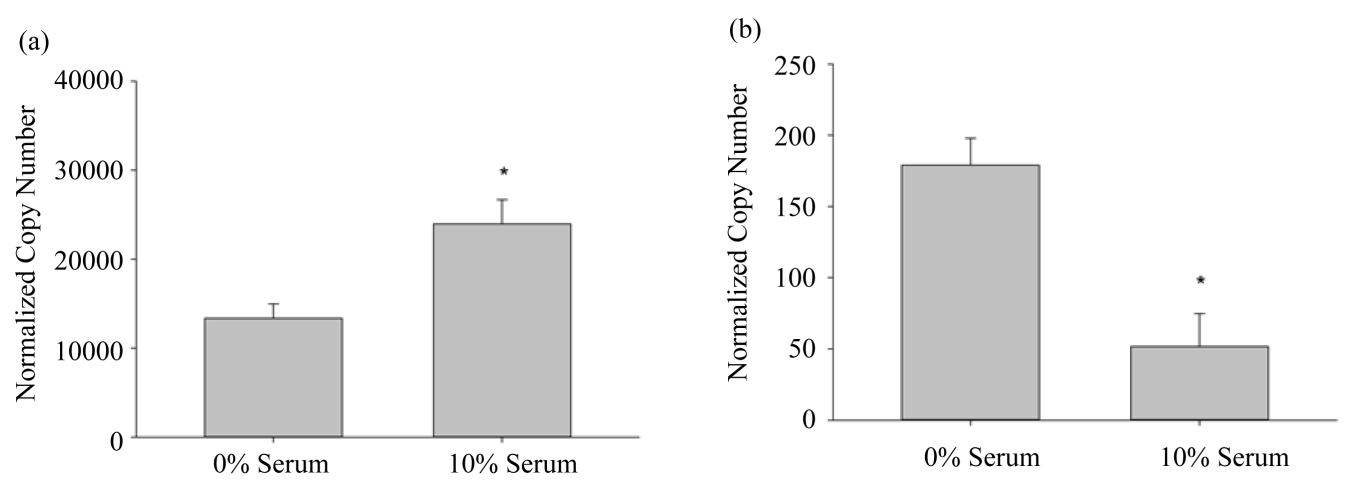

Figure 3. Differential NOS expression in pulmonary myofibroblasts. (a) NOS3 (eNOS) gene expression levels from pulmonary myofibroblasts grown in media $+/-10 \%$ serum; (b) NOS2 (iNOS) gene expression levels from pulmonary myofibroblasts grown in media $+/-10 \%$ serum. For both NOS isoforms, transcript copy number was quantified by RT-PCR and normalized to the internal control GUS, as described in Materials and Methods. Results are given as the mean copy number \pm SEM $(n=5)$; An * indicates significance $(\mathrm{p}<0.05)$ as determined by ANOVA.

(a)

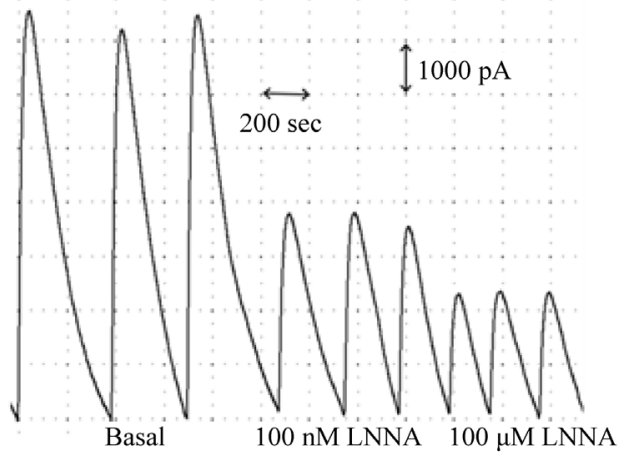

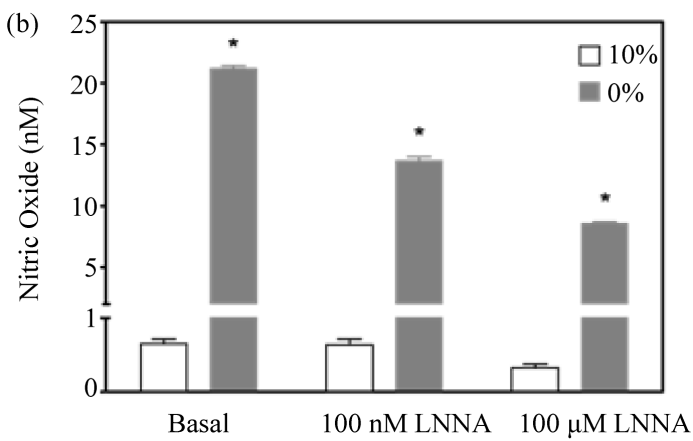

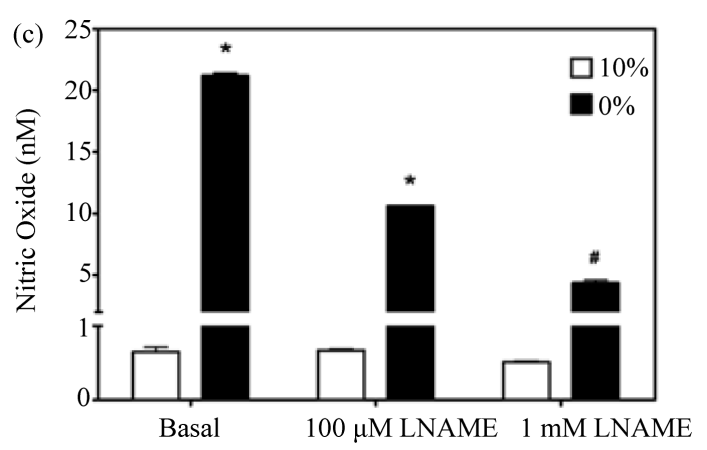

Figure 4. Dose-dependent inhibition of NO accumulation by L-NAME and L-NNA. (a) Amperogram for myofibroblast samples incubated for 3 hours in PBS +/- the non-specific NOS inhibitor, L-NNA; (b) NO produced from pulmonary myofibroblasts cultured in serum-free $(0 \%)$ or serum-supplemented $(10 \%)$ media in the presence of varying concentrations of L-NNA; (c) NO produced from pulmonary myofibroblasts cultured in serum-free $(0 \%)$ or serum-supplemented $(10 \%)$ media in the presence of varying concentrations of L-NAME. A representative dataset for three separate experiments is given. Results are given as means $\pm 1 \mathrm{SD}$. Significant differences are indicated by an * $(\mathrm{p}<0.001)$ or $\#(\mathrm{p}<0.05)$ as determined by paired $t$-test.

upon a cocktail of protein catalysts. In the current study, the very slight reduction in sensitivity ( $0.6 \mathrm{nM}$ versus $0.2 \mathrm{nM}$ reported by Boo, et al. [23]) is offset by the simplification of the nitrate reduction step. Moreover, in this study we have also refined the technique by using PBS as the assay solvent thus reducing background noise from interfering species. Matrix effects are well known in cell-based assays and are of paramount concern when measuring very low levels of analyte from cells in culture; a recent study that compared NO quantification from 
an NO-releasing film using spectrophotometric, chemiluminescent, or amperometric detection methods found that NO measured in cell culture media or serum was always significantly lower than that measured in PBS [30].

By using the assay, in conjunction with RT-PCR, we have shown for the first time that eNOS and to a lesser extent, iNOS, are the major isoforms expressed in pulmonary myofibroblasts. This expression pattern is consistent with other work in alveolar epithelial cells where the majority of NOS activity is attributable to eNOS [13]. Furthermore, we have also shown that NOS transcription and enzyme activity change as a consequence of cell differentiation. Although seemingly inconsistent at first, the observation that eNOS RNA declined in the absence of serum while its activity increased, suggests that under differentiating conditions eNOS may be more active due to its discrete subcellular location. It is well established that eNOS associates reversibly with a diverse family of proteins that regulate its trafficking in the cell and such intracellular targeting is a crucial step in regulating its activity ([31] and references therein). Moreover, the observed differentiation-dependent fluctuations in NO likely implicates this signaling pathway in regulating the proto-myofibroblast to myofibroblast transition since in other cell types NO is known to be an important inhibitor of epithelial-mesenchymal transitions [13].

Overall, the data presented in this study confirms that the refined electrode-based NOx assay is robust, reproducible, and can be used to quantify subtle changes in NO produced from constitutive NOS in pulmonary myofibroblasts. The simplicity and efficiency of the assay will allow us to continue investigating NO signaling in pulmonary myofibroblasts in culture since eNOS in vitro rates of NO production mirror physiological concentrations [19].

\section{Acknowledgements}

The project was supported in part by NIH Grants 2P20RR-16481from the IDeA Networks of Biomedical Research Excellence (INBRE) Program of the National Center for Research Resources and R15HL087185 from the NHLBI to N.A.R.

\section{References}

[1] Gabbiani, G. (1992) The Biology of the Myofibroblast. Kidney International, 41, 530-532. http://dx.doi.org/10.1038/ki.1992.75

[2] Powell, D.W., et al. (1999) Myofibroblasts. I. Paracrine Cells Important in Health and Disease. American Journal of Physiology, 277, C1-C9. http://dx.doi.org/10.1111/j.1469-7793.1999.001af.x

[3] Phan, S.H. (2002) The Myofibroblast in Pulmonary Fibrosis. Chest, 122, 286S-289S. http://dx.doi.org/10.1378/chest.122.6_suppl.286S

[4] Gabbiani, G. (1999) Some Historical and Philosophical Reflections on the Myofibroblast Concept. Current Topics in Pathology, 93, 1-5. http://dx.doi.org/10.1007/978-3-642-58456-5_1

[5] Gabbiani, G. (1996) The Cellular Derivation and the Life Span of the Myofibroblast. Pathology—Research and Practice, 192, 708-711. http://dx.doi.org/10.1016/S0344-0338(96)80092-6

[6] Schmitt-Graff, A., Desmouliere, A. and Gabbiani, G. (1994) Heterogeneity of Myofibroblast Phenotypic Features: An Example of Fibroblastic Cell Plasticity. Virchows Archiv, 425, 3-24. http://dx.doi.org/10.1007/BF00193944

[7] Schurch, W., Seemayer, T.A. and Gabbiani, G. (1998) The Myofibroblast: A Quarter Century after Its Discovery. The American Journal of Surgical Pathology, 22, 141-147. http://dx.doi.org/10.1097/00000478-199802000-00001

[8] Gentiloni Silveri, N., Mazzone, M., Portale, G. and Carbone, L. (2001) Nitric Oxide. A General Review about the Different Roles of This Innocent Radical. Minerva Medica, 92, 167-171.

[9] Moncada, S. and Higgs, A. (1993) The L-Arginine-Nitric Oxide Pathway. The New England Journal of Medicine, 329, 2002-2012. http://dx.doi.org/10.1056/NEJM199312303292706

[10] Knowles, R.G. and Moncada, S. (1994) Nitric Oxide Synthases in Mammals. Biochemical Journal, 298, 249-58.

[11] Forstermann, U., Boissel, J.P. and Kleinert, H. (1998) Expressional Control of the "Constitutive” Isoforms of Nitric Oxide Synthase (NOS I and NOS III). The FASEB Journal, 12, 773-790.

[12] Arnal, J.F., Dinh-Xuan, A.T., Pueyo, M., Darblade, B. and Rami, J. (1999) Endothelium-Derived Nitric Oxide and Vascular Physiology and Pathology. Cellular and Molecular Life Sciences, 55, 1078-1087. http://dx.doi.org/10.1007/s000180050358

[13] Vyas-Read, S., Shaul, P.W., Yuhanna, I.S. and Willis, B.C. (2007) Nitric Oxide Attenuates Epithelial-Mesenchymal Transition in Alveolar Epithelial Cells. American Journal of Physiology—Lung Cellular and Molecular Physiology, 
293, L212-L221. http://dx.doi.org/10.1152/ajplung.00475.2006

[14] Kordes, C., Sawitza, I., Müller-Marbach, A., Ale-Agha, N., Keitel, V., Klonowski-Stumpe, H. and Häussinger, D. (2007) CD133+ Hepatic Stellate Cells Are Progenitor Cells. Biochem. Biochemical and Biophysical Research Communications, 352, 410-417. http://dx.doi.org/10.1016/j.bbrc.2006.11.029

[15] Mookerjee, I., Hewitson, T.D., Halls, M.L., Summers, R.J., Mathai, M.L., Bathgate, R.A., Tregear, G.W. and Samuel, C.S. (2009) Relaxin Inhibits Renal Myofibroblast Differentiation via RXFP1, the Nitric Oxide Pathway, and Smad2. The FASEB Journal, 23, 1219-1229. http://dx.doi.org/10.1096/fj.08-120857

[16] Lu, L., Chen, S.S., Hassid, A. and Sun, Y. (2008) Cardiac Fibrogenesis Following Infarction in Mice with Deletion of Inducible Nitric Oxide Synthase. American Journal of the Medical Sciences, 335, 431-438. http://dx.doi.org/10.1097/MAJ.0b013e3181571f97

[17] Pessanh, M.G. and Mandarim-de-Lacerda, C.A. (2000) Myofibroblast Accumulation in Healing Rat Myocardium Due to Long-Term Low-Dosage Nitric Oxide Synthesis Inhibition. Experimental and Toxicologic Pathology, 52, 192-194. http://dx.doi.org/10.1016/S0940-2993(00)80027-5

[18] Gonzalez-Cadavid, N.F. and Rajfer, J. (2009) Experimental Models of Peyronie's Disease. Implications for New Therapies. Journal of Sexual Medicine, 6, 303-313. http://dx.doi.org/10.1111/j.1743-6109.2008.01104.x

[19] Hall, C.N. and Garthwaite, J. (2009) What Is the Real Physiological NO Concentration in Vivo? Nitric Oxide, 21, 92103. http://dx.doi.org/10.1016/j.niox.2009.07.002

[20] Taha, Z.H. (2003) Nitric Oxide Measurements in Biological Samples. Talanta, 61, 3-10. http://dx.doi.org/10.1016/S0039-9140(03)00354-0

[21] Räthel, T.R., Leikert, J., Vollmar, A.M. and Dirsch, V.M. (2003) Application of 4,5-Diaminofluorescein to Reliably Measure Nitric Oxide Released from Endothelial Cells in Vitro. Biological Procedures Online, 5, 136-142. http://dx.doi.org/10.1251/bpo55

[22] Leikert, J.F., Räthel, T.R., Müller, C., Vollmar, A.M. and Dirsch, V.M. (2001) Reliable in Vitro Measurement of Nitric Oxide Released from Endothelial Cells Using Low Concentrations of the Fluorescent Probe 4,5-Diaminofluorescein. FEBS Letters, 506, 131-134. http://dx.doi.org/10.1016/S0014-5793(01)02901-5

[23] Boo, Y.C., Tressel, S.L. and Jo, H. (2007) An Improved Method to Measure Nitrate/Nitrite with an NO-Selective Electrochemical Sensor. Nitric Oxide, 16, 306-312. http://dx.doi.org/10.1016/j.niox.2006.09.004

[24] Berkels, R.S., Purol-Schnabel, S. and Roesen, R. (2001) A New Method to Measure Nitrate/Nitrite with a NO-Sensitive Electrode. Journal of Applied Physiology, 90, 317-320.

[25] McIntosh, J.C., Hagood, J.S., Richardson, T.L. and Simecka, J.W. (1994) Thy1 (+) and (-) Lung Fibrosis Subpopulations in LEW and F344 Rats. European Respiratory Journal, 7, 2131-2138. http://dx.doi.org/10.1183/09031936.94.07122131

[26] Grinnell, F. (1994) Fibroblasts, Myofibroblasts, and Wound Contraction. Journal of Cell Biology, 124, 401-404. http://dx.doi.org/10.1083/jcb.124.4.401

[27] Rice, N.A. and Leinwand, L.A. (2003) Skeletal Myosin Heavy Chain Function in Cultured Lung Myofibroblasts. Journal of Cell Biology, 163, 119-129. http://dx.doi.org/10.1083/jcb.200303194

[28] Griffith, O.W. and Kilbourn, R.G. (1996) Nitric Oxide Synthase Inhibitors: Amino Acids. Methods in Enzymology, 268, 375-392. http://dx.doi.org/10.1016/S0076-6879(96)68040-9

[29] Jagnandan, D., Sessa, W.C. and Fulton, D. (2005) Intracellular Location Regulates Calcium-Calmodulin Dependent Activation of Organelle-Restricted eNOS. American Journal of Physiology: Cell Physiology, 289, C1024-C1033. http://dx.doi.org/10.1152/ajpcell.00162.2005

[30] Hunter, R.A., Storm, W.L., Coneski, P.N. and Schoenfisch, M.H. (2013) Inaccuracies of Nitric Oxide Measurement Methods in Biological Media. Analytical Chemistry, 85, 1957-1963. http://dx.doi.org/10.1021/ac303787p

[31] Dudzinski, D. and Michel, T. (2007) Life History of eNOS: Partners and Pathways. Cardiovascular Research, 75, 247260. http://dx.doi.org/10.1016/j.cardiores.2007.03.023 